\title{
High Resolution Imaging of Short-Range Order Materials (Allophane) with Aberration Corrected TEM and Direct Electron Detection
}

\author{
Thomas G. Sharp ${ }^{1,2}$ and Shery L.Y.Chang ${ }^{2}$ \\ ${ }^{1 .}$ School of Earth and Space Exploration, Arizona State University, Tempe AZ, USA \\ 2. LeRoy Eyring Center For Solid State Science, Arizona State University, Tempe AZ, USA
}

\begin{abstract}
Aberration-corrected TEM and STEM instrumentation can provide atomic-scale imaging and chemical analysis if the structures are not damaged by the high electron doses used to obtain such images. This can be especially problematic for some geologic materials such as zeolites and other framework silicates, high-pressure minerals and short-range ordered (SRO) materials. Here we focus on imaging the SRO, allophane.
\end{abstract}

Allophane is a poorly crystalline hydrous aluminosilicate that is common in volcanic soils. It is a product of low-temperature aqueous alteration of feldspars and volcanic glasses [1]. It was suspected on the surface of Mars [2], where mid-IR spectroscopy indicated abundant amorphous silicate and lowtemperature chemical weathering is likely. Rampe et al. [3] used synthetic allophanes and mid IR spectroscopy to demonstrate that much of the amorphous surface component of Martian soils is likely allophane. As part of that study, TEM was used to characterize a range of allophane compositions. Allophanes are thought to consist of 4-5 nm hollow spheres with a gibbsite-like $\mathrm{Al}(\mathrm{OH})_{3}$ outer layer enclosing non-polymerized silicate. Our previous efforts to resolve these structures with conventional HRTEM have produced poor quality image as a result of rapid beam damage (Figure 1). The purpose of this study is to explore the use of aberration corrected TEM combined with a direct detection camera to observe atomic structure in SRO materials.

Synthetic allophanes and aluminosilicate gels in four compositions ( $\mathrm{Si} / \mathrm{Al}$ ratios of 0.44, 0.58, 0.92 and 3) [3] were imaged with a Philips CM200-FEG at $200 \mathrm{kV}$. These samples were later imaged with the FEI Titan aberration corrected ETEM with an X-FEG monochromatic electron source. The imagecorrected Titan has a Gatan K2 Summit direct detection camera (DDC) on loan for application experiments. The Titan was run at $300 \mathrm{kV}$, using the monochrometer and corrector. The K2 Summit DDC was run both in counting mode and in linear mode.

Low-magnification imaging of the various allophane compositions with the CM200 showed that most of the material consists of micron-sized clumps of 5 to $30 \mathrm{~nm}$ nanoparticles (Figure 1). Some of the samples were less porous and appeared to be fragments rather than fluffy aggregates of nanoparticles. Attempts at high-resolution imaging of the nanoparticle structures failed because of irradiation-induced damage that caused the particle clusters to deform, resulting in rapid sample drift. The nanoparticles also lost contrast and changed shape, indicating damage and loss of short-range order.

Images from the FEI Titan with the $\mathrm{K} 2 \mathrm{DDC}$ on $(\mathrm{Si} / \mathrm{Al}=3)$ allophane were taken form the same material as shown in Figure $1 \mathrm{~b}$ and $\mathrm{c}$. This sample does not have the nano-particulate structure seen in other samples. Instead, we observed a gel-like fragment without evidence of spheroidal structures. Images taken in counting mode with a dose rate of $\sim 12$ electrons/pixel/second (eps) showed almost no observable beam damage and very little sample drift. Images taken in linear mode with dose rates of up to 1000 eps also resulted in relatively little beam damage. The only SRO structures observed were clay 
nanocrystals (Figure 2). We imaged one such particle in counting mode, integrating ten exposures of 0.2 seconds with drift correction. This approach produced nearly atomic resolution of the clay structure (Figure 2) with no apparent beam damage. Further analysis of the data is needed to interpret the structural details. These initial results indicate that this approach will allow for much improved imaging of structural details in SRO materials. These results suggest that many beam-sensitive materials silicates will be suitable for atomic scale imaging.

\section{References:}

[1] S. M. Coleman, U.S. Geological Survey Professional Paper 1246 (1981), p. 51.

[2] J. R. Michalski et al, Icarus 174 (2004), p. 161.

[3] E. B. Rampe et al, Geology (2012) p. 995.

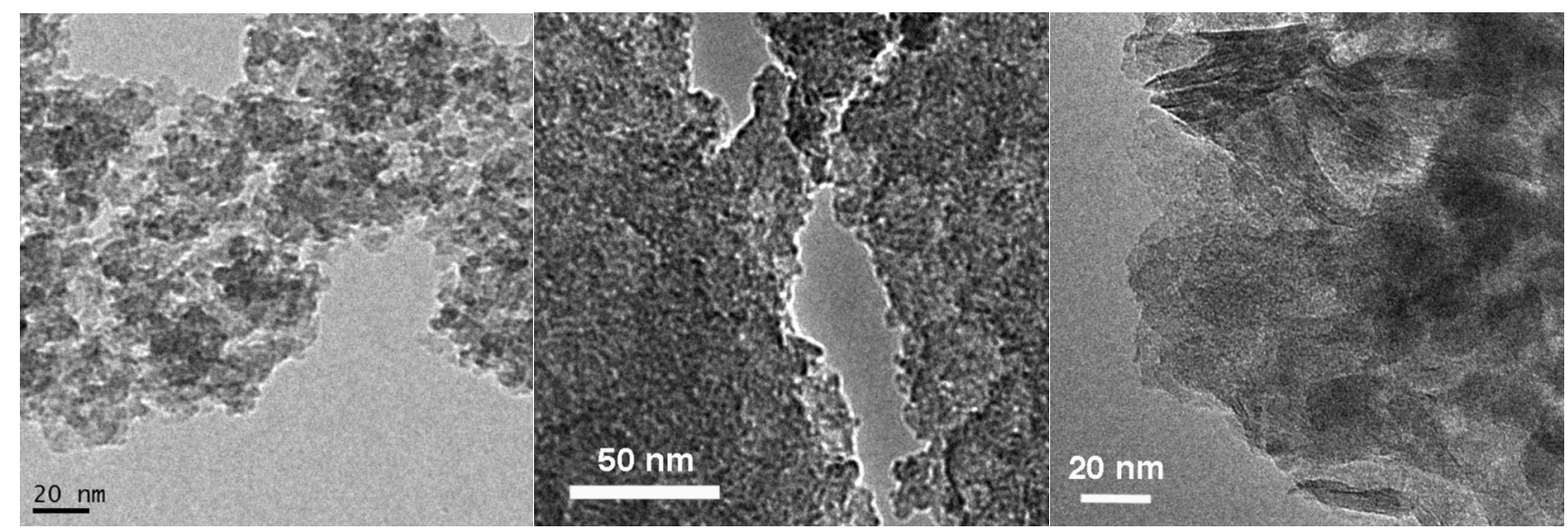

Figure 1. (a) bright-field image of particulate allophane. (b) bright-field image of non-particulate allophane showing nano-scale granular texture. (c) Higher magnification image of sample shown in (b) showing effects of beam damage and sample drift.

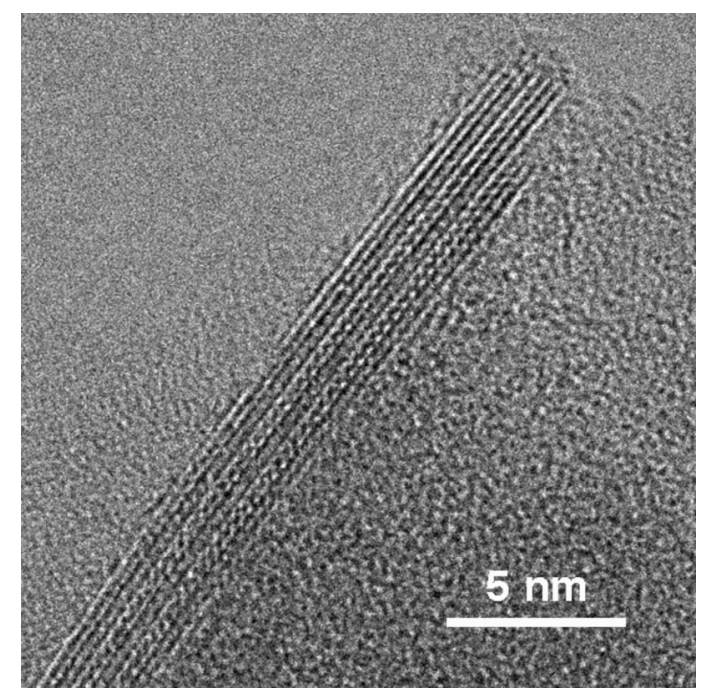

Figure 2. HRTEM image of a clay nano-crystal within the same allophane sample as Fig $1 \mathrm{~b}$ and c. Ten counting mode images were integrated after correcting for minor sample drift. Detailed structure within the clay layers is clearly visible. 